\title{
Multi-level Social Networking to Enable and Foster Collaborative Organizations
}

\author{
Youakim Badr ${ }^{1}$, Noura Faci $^{2}$, Zakaria Maamar ${ }^{3}$, and Frédérique Biennier ${ }^{1}$ \\ ${ }^{1}$ INSA de Lyon, F-69621, Villeurbanne, France \\ ${ }^{2}$ Université Lyon 1, F-69622, Villeurbanne, France \\ ${ }^{3}$ Zayed University, U.A.E. \\ \{youakim.badr, frederique.biennier\}@insa-lyon.fr, \\ noura.faci@univ-lyon1.fr, zakaria.maamar@zu.ac.ae
}

\begin{abstract}
Recent interest in capitalizing on the social network phenomenon has shown some relatively successful benefit to the business world. Social networking can sustain large-scale collaborative strategies between people and organizations. However, the main challenge remains the smooth weaving of social networks into organizations daily operations. In this paper we extend the reach and scope of social networks for an entire organization and show how they provide benefit to the business as a whole. We introduce a multi-layered social network model where people may play simultaneously one or more roles (e.g., suppliers, competitors, and customers) and participate in providing products, supporting services, and/or consuming them in sustainable collaborative environments. The experiments demonstrate both the widespread interest and possibilities of online social communities to foster collaboration among organizations.
\end{abstract}

Keywords: Social network, collaboration, business model, virtual organization.

\section{Introduction}

Web 2.0, one of the latest buzzwords in information and communication technologies domain, encourages people to break the isolation barrier so they can engage over the Internet in various activities that usually lead into creating knowledge (e.g., blogs and wikis), sharing information (e.g., mashups and folksonomies), and establishing contacts (e.g., social networking sites). Capitalizing on people's positive experiences about Web 2.0, many organizations consider that improving their product quality and service delivery can, also, happen through Web 2.0. Employees are these days invited to embrace social networks such as Facebook to establish and foster contacts with their colleagues, customers, and suppliers. Organizations aim at streamlining their business processes with their strategic plans and adapting their best practices in response to the opportunities that these applications offer [2]. Gearing organizationflavored social networks towards the specific needs of customers, suppliers, partners, and competitors organizations look forward to establishing new partnerships with their different stakeholders to effectively undertake joint business activities including resource co-design, co-marketing, co-production, co-development, and sharing. These 
new business conditions lead in significant structure and behavior changes within and cross organizations, such as focusing on core processes and promoting interoperability. To support organizations in their new endeavors, one can think of taking advantage of Internet-based social networks to build "trusted business communities". In these communities, people with different backgrounds and from different locations collaborate to co-create new value-added services. In addition, each member of a business community may play simultaneously one or more roles (e.g., provider, worker, and customer) and participate in providing products, supporting services and/or consuming them in sustainable and economical environments. This human-centric community leads in the development of human networks, spanning across organizational boundaries.

After presenting the background and motivation in section 2, we propose a multilayer social network model to design a collaborative organization in section 3 . The capitalized collaborative relationships are illustrated in the experimental results section and used to facilitate and enrich business collaboration to co-create new services and / or improve the partnership / service discovery and adaptation processes. Weaving social networks into the organization information systems will open up new business opportunities and transform organizations into socio-technical ecosystems driven by collaboration and information exchange.

\section{Background}

Due to market trends and fast-changing conditions, organizations should be more flexible, adapt to business changes and increase customer satisfaction, leading in new product-service collaborative organizations [3]. This induces significant organizational and strategic adaptations within organizations, such as focusing on their core business and looking for new collaboration strategies. Such business strategies raise the importance of developing outsourcing policies and promoting inter-enterprise collaborative business leading to alliances or network-based organizations. Several works have addressed the way collaborative organizations are set, paying attention on the firms collaboration "maturity" leading to set "collaborative breeding environments" gathering firms that "could collaborate" or paying more attention on trust and personal relationships factors to set collaborative trusted communities [10].

Roughly speaking, networked collaborative organizations consist of nodes and edges. The nodes refer to any type of object or entity such as individuals or organizations, and the edges refer to relationships between these nodes such as the degree of friendship between two persons or distance between two cities. Relationships are sometimes directional, bidirectional, with weight, or a mixture of all of these. Social networks are known to be tacit, informal, and intangible. This can hinder their identification and proper use. In previous work [1] [2], we focused on the importance of acknowledging their existence in organizations, so they can be structured to become formal and tangible representations. In an organization, we have identified four types of inter-related social networks [1]: customer, supplier, competitor, and partner. 
1. Customer-centric social network states the nature of relationships between an enterprise's customer and the acquaintances of this customer in the network, such as friendship and kinship.

2. Supplier-centric social network states the nature of relationships between an enterprise's supplier and the acquaintances of this supplier in the network, such as supplier-of-supplier and competition.

3. Competitor-centric social network states the nature of relationships between an enterprise's competitor and the acquaintances of this competitor in the network, such as spontaneous-collaboration and competitor-of-competitor.

4. Finally, partner-centric social network states the nature of relationships between an enterprise's partner and the acquaintances of this partner in the network such as add-on and alliance.

These relationships bring to light the importance of corporate social networking. The use of social networks is reported in different domains such as social sciences, artificial intelligence, and business sciences. Ethier notes that "the study of social networks is important since it helps us to better understand how and why we interact with each other, as well as how technology can alter this interaction. The field of social network theory has grown considerably during the past few years as advanced computing technology has opened the door for new research" [4]. Raab and Milward note that social networks are either bright or dark [5]. The former occur when the outcomes of socializing turn out beneficial for individuals, groups, businesses, and society at large. Contrarily, the latter occur when the outcomes of socializing are obtained at great cost to individuals, groups, businesses, and social welfare. Research reveals that social networks operate at many levels, from families up to the level of nations, and play a role in determining first, how problems are solved and organizations are run, and second, the degree to which individuals succeed in achieving their goals. Facebook, MySpace, and LinkedIn offer a glimpse of today's social applications that help people deploy and manage their own Web-based social networks. Social networking brings a new dimension to the Web by offering novel communication channels between people and between communities [7]. Connolly states that "since social Web applications are built to encourage communication between people, they typically emphasize some combination of the following social attributes: identity, reputation, presence, relationships, groups, conversations, and sharing" [8].

\section{Towards Social Organizations}

To deal with the large-scale collaboration challenge among organizations, we propose to take advantage of social networking to discover potential partners to fit new business opportunities. This involves coupling the functional and non functional description of business or industrial services offered by the different firms including, for example, partner profile and competencies, product offer, industrial and business process specifications to corporate social networks so that business or industrial offers can be discovered, selected, and composed to set collaborative organizations [9]. This selection process integrates both "production knowledge", namely partner competencies and capabilities, environmental constraints such as trusted environment, 
IT support interoperability, sustainability and partner, and "social behaviour" in order to capitalize partner's collaborative activities and to evaluate partner's ability to set successful collaboration. It is for sure that embracing social networks in the world of work would raise important human issues such as privacy, confidentiality and trustworthiness requiring a legal framework to address these issues as well as new community models to integrate corporate and business knowledge.

To deal with this challenge, we define a multi-level social network model as a hyper graph showing the connection between employees, customers, suppliers, partners, and competitors. Participants in a multi-level social network connect to each other through different relationships representing multiple types of networks overlaid on top of one another. Each relationship, which is ranging from information sharing to social bindings, refers to a particular role played by the participants.

\subsection{Value-Added of Social Networks to Organizations}

We model the multi-layered social network as a hyper graph structure with $d$-layers: where the first layer is the physical network and each layer connects subset of nodes playing same role and binding to various relationships. As an example, we focus on a multi-layered social networks consisting of customers, suppliers, competitors, and partners. These social networks should be woven into the organization of day-to-day operations so that socio-economical factors as competitiveness level between customers and fairness selection of providers are taken into account. Each social network has a value-added to this operation implementation as follows (Fig. 1):

1. Customer social network permits to identify potential relationships between customers such as competition, referral, and collaboration so that new business opportunities can be identified by the enterprises to fit better the customer needs and improve its competitiveness level:

- Competition relationship. captures the number of times that a group of customers had to compete over specific products and services offered by the organization and the customers who won the competitions. This should allow the organization to guarantee a fair competition by strengthening its ability to identify appropriate business opportunities with the whole of its customers like adjusting the nature of products and services to offer in response to these customers' demands.

- Referral relationship. captures the number of times that a group of customers dealt with the organization based on the recommendation of other customers and how relevant these recommendations were for the organization. This should allow the organization identify business opportunities in response to customers' expectations.

- Collaboration relationship. captures the number of times that a group of customers got together to form collective groups when requesting products and services from the organization and how fruitful these collaborations were for the organization. This should enable the organization increase its productivity and benefits and as a result improve its competiveness level.

2. Supplier social network permits to identify potential relationships between suppliers: 
- Competition relationship captures the number of times that a group of suppliers had to compete over specific calls for proposals generated by the organization and the suppliers that won the competitions. This should enable the organization exploit better the economic value that suppliers can provide by adjusting the calls for proposals.

- Referral relationship captures the number of times that a group of suppliers dealt with the organization based on the recommendation of other suppliers when they cannot participate in a competition and how relevant the recommendations were for the organization. Therefore, suppliers can recommend other suppliers for keeping their competitiveness level with their respective competitors. Based on past recommendation experiences, the organizations can trust or not their suppliers.

- Collaboration relationship captures the number of times that a group of suppliers got together when providing the products for the suppliers of the organization. This should enable the organization guarantee fruitful collaboration by identifying the suppliers of suppliers to solicit.

3. Partner social network captures partnerships experiences:

- Collaboration relationship captures the number of times a partner provides the customers with satisfactory services. This should allow the organization secure the loyalty of the customers.

- Referral relationship captures the number of times a group of partners support the organization based on the recommendations of other partners when they cannot participate in collaborations and how relevant the recommendations were for the organization.

4. Competitor social network permits to identify potential relationships between the organization's competitors such as competition and referral.

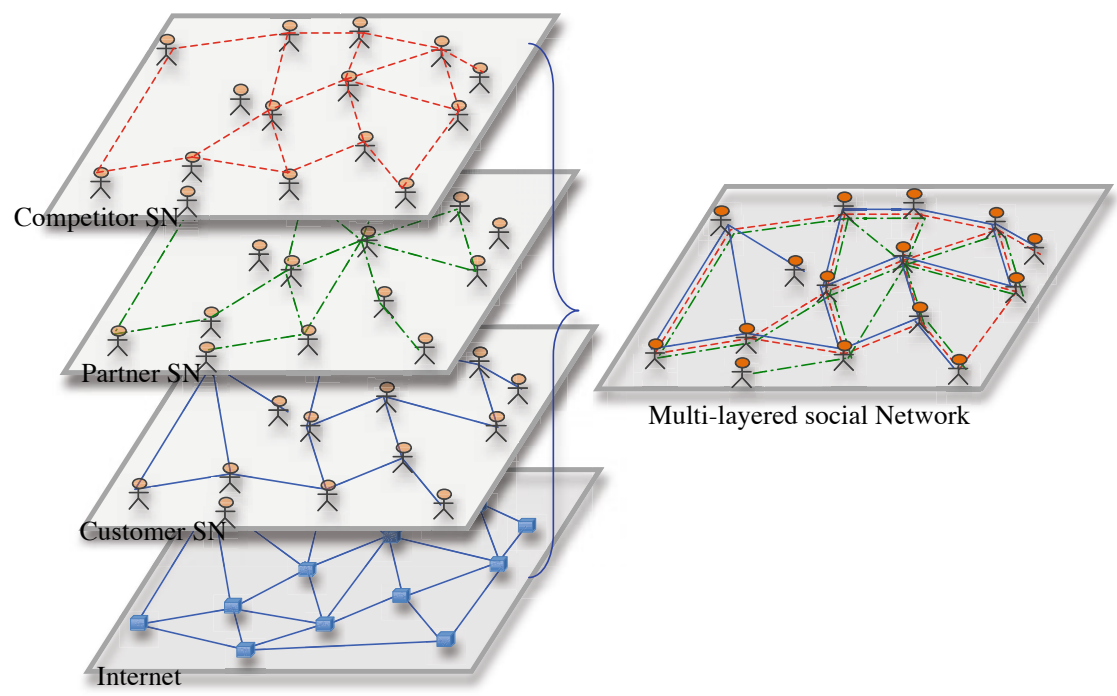

Fig. 1. A Multi-layered Social Network including Competitors, Partners and Customers 
- Competition relationship captures the number of times that the organization and a group of competitors compete in global marketplace and who won the competitions. This should enable the organization improve its competitiveness level by adjusting the Partner-centric SN and Supplier-centric SN (e.g., add new partners/suppliers and release old ones).

- Referral relationship captures the number of times that a group of competitors compete with the organization based on the recommendation of other competitors when they cannot participate in competition and how negative the recommendations were to the organization.3.2. Formal Representation of the Multi-layered Social Network.

We define the multi-layered social network, $M$, of a given Organisation, $O$, as a tuple (Nodes, Roles, SocialRelationships), where:

- Nodes denotes the set of nodes constituting the whole social community of $O$ and $n$ the cardinality of Nodes.

- Roles denotes the set of available roles the nodes can play and $r$ the cardinality of Roles. Each role defines a particular layer in the multi-layered social network among any subset of Nodes. For example, an organisation can build its social community by focusing on specific roles such as customers, suppliers, competitors, and partners just to mention. In this case, Roles $=$ \{customer, supplier, competitor, partner $\}$. We also denote by $P_{\text {role }} \in$ Roles the set of all possible relationships among nodes of role and $m$ the cardinality of $P_{\text {role }}$. For example, an organisation may focus on relationships, such as competition, referral and collaboration with its customers. In this case, the relationship set is defined as $P_{\text {customer }}=\{$ competition, referral, collaboration .

- SocialRelationships denotes the matrix of social relationships for any node with respect to all its peers and all its possible roles. The matrix has $(n \times n \times \mathrm{x} r)$ dimension. We assume that the matrix ( $n \times n \times 1)$ defines a basic physical network $I$, such as Internet, connecting all nodes by means of information and communication technologies. Each element $\mathrm{w}_{\mathrm{ijk}}$ of the matrix is a $\mathrm{t}$-uple $<\mathrm{V}^{1}, \mathrm{~V}^{2}$, $\ldots, \mathrm{V}^{\mathrm{m}}>_{\mathrm{ijk}}$ where $\mathrm{V}^{\mathrm{q}}$ is the quantitative value associated with the $\mathrm{q}^{\text {th }}$ social relationship in $\mathrm{P}_{\mathrm{k}}$ between the nodes $i$ and $j$ playing the role $k$. In order to normalize the matrix, we introduce $\mathrm{T}^{\mathrm{q}}{ }_{\mathrm{ik}}$ is the total number of times that the node $i$, playing the role $k$, has participated in the $\mathrm{q}^{\text {th }}$ social relationship.

To update $\mathrm{w}_{\mathrm{ijk}}$, appropriate functions are suggested per type of social relationship and type of social network. For example, the function to evaluate $\mathrm{w}_{\mathrm{ijk}}$ in a customercentric social network can be given by the following formulas:

- The value $\mathrm{V}_{\mathrm{i}, \mathrm{j}}^{\text {competition }}$ of the competition relationship between a group of customers $\mathrm{c}_{\mathrm{i}}$ and $\mathrm{c}_{\mathrm{j}}$ is computed as $\mathrm{V}_{\text {competition }}^{\text {ci, }}=\omega_{\mathrm{ij}} / \Theta_{\mathrm{ij}}$, where $\omega_{\mathrm{ij}}$ is the number of times $\mathrm{c}_{\mathrm{i}}$ won the competition against $c_{j}$, $\Theta_{i j}$ the number of times $c_{i}$ and $c_{j}$ compete, and $\mathrm{T}_{\text {ci, customer }}^{\text {competition }}=\sum_{\mathrm{l}=1, \mathrm{n}} \Theta_{\mathrm{il}}$.

- The value $\mathrm{V}^{\text {referral }}{ }_{\mathrm{i}, \mathrm{j}}$ of the referral relationship between a group of customers $c_{i}$ and $\mathrm{c}_{\mathrm{j}}$ is computed as $\mathrm{V}_{\mathrm{c} \text { refer } \mathrm{j}}=\mathrm{O}_{\mathrm{ij}} * \sigma_{\mathrm{ij}}$, where $\mathrm{O}_{\mathrm{ij}}$ is the relevance factor given by the organization in terms of subsequent business opportunities, $\sigma_{\mathrm{ij}}$ is the number of times $\mathrm{c}_{\mathrm{i}}$ recommends $\mathrm{c}_{\mathrm{j}}$, and $\mathrm{T}_{\mathrm{ci} \text {, customer }}^{\text {referral }}=\sum_{\mathrm{l}=1, \mathrm{n}} \sigma_{\mathrm{il}}$. 
- The value $\mathrm{V}_{\mathrm{i}, \mathrm{j}}^{\text {collaboration }}$ of the collaboration relationship between a group of customers $\mathrm{c}_{\mathrm{i}}$ and $\mathrm{c}_{\mathrm{j}}$ is computed as $\mathrm{V}^{\text {collaboration }}{ }_{\mathrm{ci}, \mathrm{cj}}=\Phi_{\mathrm{ij}} * \pi_{\mathrm{ij}}$, where $\Phi_{\mathrm{ij}}$ is the fruitfulness factor given by the organization in terms of productivity and $\pi_{\mathrm{ij}}$ the number of times $\mathrm{c}_{\mathrm{i}}$ collaborates with $\mathrm{c}_{\mathrm{j}}$, and $\mathrm{T}_{\mathrm{ci} \text {, customer }}^{\text {collaboration }}=\sum_{\mathrm{l}=1, \mathrm{n}} \pi_{\mathrm{i}}$.

\section{Experimental Results}

To measure the value-added of multi-layered social networks to an organization, we apply social network analysis to measure interactions between people. We consider a real case study related to advertising of a new perfume. The marketing department leads the study by contacting perfume retailers and negotiating with their customers exhibition stands, discount rates and whether they accept to recommend the new perfume to their clients by sending them messages on twitter. These "digital community relationships" are used to build the user centric community model: each node in our model is associated to an user identified by his/her email address and We build our social data from customer emails, twitter usernames respectively. However, edges reflect exchange between users, either by twitter, such as if available, and a twitter hash tag to mark the new perfume and categorize its related messages in a Tweet or by email to identify collaborations between users. We also exploit the open source software Gephi [6] to visualize our customer social networks respectively with respect to the collaboration relationship based on exchanged emails and the referral relationship based on twit messages. In addition, we use the spigot plug in to analyze email contacts with customers. Figure 2 (left) displays the collaboration relationship network as a directed acyclic graph of 332 nodes and 693 edges. Moreover, the spigot plug in allows us to built a network about customers who follow each other and who search or mention the new perfume hash tag. Figure 2 (right) displays a network based on the referral relationship of customers who twit the new perfume. The network is a directed graph of 174 nodes and 590 connections. Since the marketing department has a list of customer emails and their twitter usernames, it becomes possible to study the customer social network with respect to collaboration and recommendation relationships and evaluate their impact on social network metrics (Average Path Length, Average Degree, Network Diameter, Graph Density, etc.).
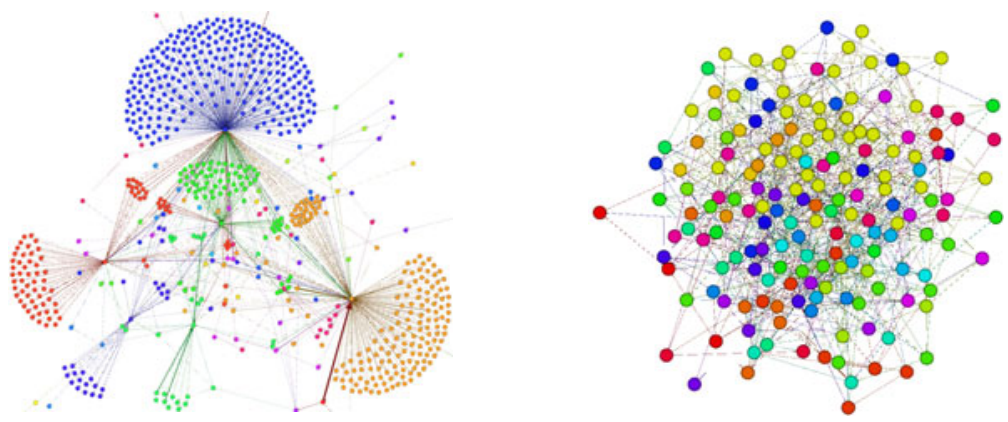

Fig. 2. Customer social network based on collaboration and referral relationships 


\section{Conclusion}

In this paper we presented a multi-layered social-network-based architecture to support business collaboration. This architecture consists of four layers referring to social networks that respectively include consumers, providers, partners and competitors of an organization and ensure their connection through different relationships like collaboration, competition, and referral. The value-added of each social network to organizations was also discussed per layer. The implementation of "this model was illustrated with customers social networks based on twitter and emails through and based on collaboration and referral relationships. The experimental results show customers' behavior during collaboration and their influence on each other.

Acknowledgement . This work is a part of the project PROCESS 2.0 (2010-2012) and generously funded by the "Direction Générale de la Compétitivité, de l'Industrie et de Services" of the French Ministry of Economy and supported by the MidiPyrénées Region.

\section{References}

[1] Badr, Y., Maamar, Z.: Can Enterprises Capitalize on their Social Networks? The Cutter IT Journal, Issue on Measuring the Success of Social Networks 22(10) (2009)

[2] Maamar, Z., Badr, Y.: Social Networks as a Service in Modern Enterprises. In: The International Conference on the Current Trends in Information Technology (CTIT 2009), Dubai, United Arab Emirates (2009)

[3] Elhabib, N., Boucher, X., Peillon, S.: Collaborative Networks for a sustainable world. In: Camarinha-Matos, L.M., Afsarmanesh, H., Boucher, X. (eds.) PRO-VE 2010. IFIP AICT, vol. 336. Springer, Heidelberg (2010)

[4] Ethier, J.: Current Research in Social Network Theory,

http://www.ccs.neu.edu/home/perrolle/archive/EthierSocialNetworks.html (visited September 2008)

[5] Raab, J., Milward, H.B.: Dark Networks as Problems. Journal of Public Administration Research and Theory 13(4) (2003)

[6] Bastian, M., Heymann, S., Jacomy, M.: Gephi: An Open Source Software for Exploring and Manipulating Networks. In: The International AAAI Conference on Weblogs and Social Media (2009)

[7] Vossen, G.: Web 2.0: A Buzzword, a Serious Development, Just Fun, or What? In: Proceedings of the International Conference on Security and Cryptography (SECRYPT 2009), Milan, Italy (2009)

[8] Connolly, S.: 7 Key Attributes of Social Web Applications, http: //connollyshaun.blogspot.com/2008/05/7-keyattributesof-social-web.html (visited April 2010)

[9] Biennier, F., Aubry, R., Maranzana, M.: Integration of Business and Industrial Knowledge on Services to Set Trusted Business Communities of Organisations. In: Camarinha-Matos, L.M., Boucher, X., Afsarmanesh, H. (eds.) PRO-VE 2010. IFIP AICT, vol. 336, pp. 420-426. Springer, Heidelberg (2010)

[10] Afsarmanesh, H., Camarinha-Matos, L.M.: A Framework for Management of Virtual Organizations Breeding Environments. In: Proceedings of PRO-VE 2005 - Collaborative Networks and their Breeding Environments, Valencia, Spain, September 26-28, pp. 35 48. Springer, Boston (2005) 